\title{
Left Atrial and Pulmonary Vein Ostial Ablation as a New Treatment for Curing Persistent Atrial Fibrillation
} Initial Experience and Results

\author{
Hiroshi Tada, MD; Kenji Kurosaki, MD; Sachiko Ito, MD; Shigeto Naito, MD; \\ Minoru Yamada, MD; Kohei Miyaji, MD; Tohru Hashimoto, MD; \\ Yuko Yoshimura, MD; Akihiko Nogami, MD*; \\ Shigeru Oshima, MD; Koichi Taniguchi, MD
}

\begin{abstract}
Background Segmental pulmonary vein (PV) isolation has been performed to eliminate paroxysmal atrial fibrillation (AF). However, this technique is not effective in most patients with persistent AF.

Methods and Results Left atrial catheter ablation (LACA) was performed by encircling the left- and rightsided PV 1-2 cm from the ostia, guided by an electroanatomical mapping system in 16 patients with persistent AF (>1 month). Twelve patients (75\%) had a history of unsuccessful transthoracic cardioversion and prophylactic antiarrhythmic drugs. Ablation lines were also created in the mitral isthmus and posterior LA. PV isolation was also performed for each PV if there were residual PV potentials after the LACA. After LACA, 38 PV (59\%) were completely isolated, and complete PV isolation was achieved with only a few radiofrequency energy applications $(2.7 \pm 2.0 \mathrm{~min})$ on a narrow area of the PV ostium $(24 \pm 15 \%)$ in the remaining PV. The mean procedure

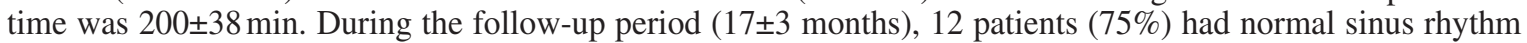
and were free of symptomatic AF with $(n=10)$ or without antiarrhythmic drugs $(n=2)$. One patient had a stroke just after the procedure. No other complications including PV narrowing $(>50 \%)$ occurred.

Conclusion This approach was effective in persistent AF, however, concomitant use of antiarrhythmic drugs was often required. (Circ J 2005; 69: 1057-1063)
\end{abstract}

Key Words: Atrial fibrillation; Catheter ablation; Left atrium; Pulmonary vein

B ecause arrhythmogenic activity that originates in the muscular sleeves of the pulmonary veins (PV) may trigger or perpetuate atrial fibrillation (AF), segmental ostial ablation to electrically isolate the PV from the left atrium (PV isolation) has been performed to eliminate paroxysmal AF (PAF) ${ }^{1-6}$ However, this technique is not effective in most patients with persistent AF or in approximately $20 \%$ to $40 \%$ of the patients with PAF $^{3-6}$ Furthermore, PV stenosis has been raised as one of the potential complications after PV isolation?,8 Recently, left atrial catheter ablation (LACA) has been reported as a more effective treatment than PV isolation for PAF, but the effectiveness of this treatment for persistent $\mathrm{AF}$ has not been sufficiently clarified?,10 Although no significant PV stenosis has occurred after LACA because of the lack of radiofrequency (RF) applications at the PV ostium, 10 esophageal fistulas have been raised as a potential complication after LACA with an 8-mm tip ablation catheter!1 Even in LACA, the recovery of the conduction between the

(Received December 27, 2004; revised manuscript received June 8, 2005; accepted June 13, 2005)

Division of Cardiology, Gunma Prefectural Cardiovascular Center, Maebashi, *Division of Cardiology, Yokohama Rosai Hospital, Yokohama, Japan

Mailing address: Hiroshi Tada, MD, Division of Cardiology, Gunma Prefectural Cardiovascular Center, 3-12 Kameizumi, Maebashi 371-

0004, Japan._E-mail: tada.h@cvc.pref.gunma.jp left atrium (LA) and PV has been considered as a dominant factor for recurrent atrial tachyarrhythmias, as is the case with PV isolation! ${ }^{12,13}$ Therefore, we felt that both a reduction in the total amount of RF energy delivered to the LA posterior wall with a large tip catheter and the completion of the PV isolation may be important in decreasing both the risk of esophageal fistulas after LACA and the AF recurrence. In a preliminary study in a patient with persistent AF, we found that the completion of the PV isolation could be achieved with only a few RF energy deliveries to a narrow area at the PV ostium in the PV with residual conduction gaps after LACA! 4

In the present study, we attempted to determine the utility of a new approach of RF catheter ablation (RFCA), which was a combined approach of RFCA of the LA and $\mathrm{PV}$ ostia, for curing persistent AF.

\section{Methods}

Study Population

The subjects in this study consisted of 16 patients with drug-refractory, persistent AF, who underwent RFCA (12 men, 4 women; mean age, $61 \pm 7$ years). The mean duration of symptomatic AF was 5.5 \pm 3.2 years, and lasted for a mean of $3.5 \pm 2.9$ years (range, $0.5-10$ years) before the ablation procedure. A mean of $2.9 \pm 1.2$ antiarrhythmic drugs had been ineffective in preventing recurrences of $\mathrm{AF}$ 

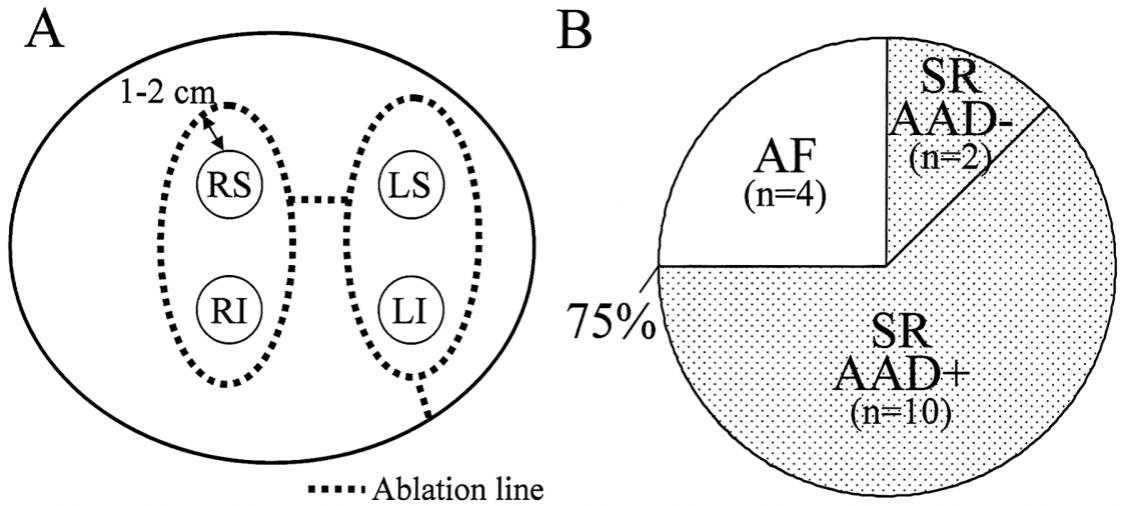

Fig 1. (A) Scheme demonstrating the left atrial catheter ablation. (B) Mid-term results of the ablation procedure in patients with persistent atrial fibrillation (AF). AAD, antiarrhythmic drug; LI(S), left inferior (superior) pulmonary vein; RI(S), right inferior (superior) pulmonary vein; SR, sinus rhythm.

Table 1 Measurement Variables of the Segmental Pulmonary Vein Isolation After Left Atrial Catheter Ablation

\begin{tabular}{lccccc}
\hline \hline & $L S P V$ & $L I P V$ & $R S P V$ & $R I P V$ & Total \\
\hline No. of PV requiring isolation after left atrial ablation & $15 / 16$ & $7 / 16$ & $11 / 16$ & $5 / 16$ & $38 / 64$ \\
Procedure time (min/PV) & $(94 \%)$ & $(44 \%)$ & $(69 \%)$ & $(31 \%)$ & $(59 \%)$ \\
No. of RF applications (/PV) & $7.7 \pm 5.1$ & $5.1 \pm 3.4$ & $10.8 \pm 12.5$ & $6.2 \pm 2.7$ & $7.7 \pm 7.7$ \\
Duration of RF energy (min/PV) & $2.5 \pm 1.9$ & $2.1 \pm 1.5$ & $3.2 \pm 2.8$ & $2.3 \pm 1.4$ & $2.7 \pm 2.0$ \\
Ablated area for isolation (\%/PV) & $2.7 \pm 1.7$ & $2.1 \pm 1.3$ & $3.1 \pm 2.9$ & $3.0 \pm 1.2$ & $2.7 \pm 2.0$ \\
No. of PV completely isolated after PV isolation (\%) & $25 \pm 16$ & $24 \pm 14$ & $25 \pm 17$ & $22 \pm 13$ & $24 \pm 15$ \\
& $15 / 16$ & $16 / 16$ & $15 / 16$ & $16 / 16$ & $62 / 64$ \\
& $(94 \%)$ & $(100 \%)$ & $(94 \%)$ & $(100 \%)$ & $(97 \%)$ \\
\hline
\end{tabular}

Values are mean \pm standard deviation. $L S(I)$, left superior (inferior); $P V$, pulmonary vein; $R S$ (I), right superior (inferior); $R F$, radiofrequency.

or restoring sinus rhythm before the ablation procedure. Twelve patients $(75 \%)$ had a history of recurrent AF after electrical cardioversion and following treatment with a prophylactic antiarrhythmic therapy. AF was considered persistent when it was present for $>30$ days and when cardioversion was required to restore sinus rhythm. Two patients had mitral stenosis, 1 primary cardiomyopathy, 1 old myocardial infarction, and the remaining 12 patients $(75 \%)$ no structural heart disease. Echocardiography demonstrated a mean left ventricular ejection fraction of $0.64 \pm 0.12$ (range, $0.41-0.84$ ), and mean LA diameter of $45 \pm 5 \mathrm{~mm}$ (range, $36-56 \mathrm{~mm}$ ).

\section{RFCA}

After informed consent was obtained, LACA was performed as previously reported? A 4-mm-tip, deflectable catheter (Navistar, Biosense Webster, Inc, Diamond Bar, CA) was introduced into the LA. A 3-dimensional shell representing the LA was constructed through the use of an electroanatomic mapping system (CARTO, BiosenseWebster). LACA was performed 1 to $2 \mathrm{~cm}$ from the PV ostia to encircle the left- and right-sided PV (Fig 1A)? However, in the presence of a relatively narrow border between the anterior aspect of the left PV and the LA appendage, ablation was performed within $5 \mathrm{~mm}$ of the ostium of the PV9,12,15 In addition to the lesions that encircled the left and right-sided PV, the 2 circumferential ablation lines were connected with an ablation line along the posterior LA. RF ablation also was performed along the mitral isthmus, between the inferior portion of the leftsided encircling lesion and the lateral mitral valve annulus. $\mathrm{RF}$ energy was delivered at a target temperature of $55^{\circ} \mathrm{C}$ and a maximum power of $35-40 \mathrm{~W}$. Ablation sites were tagged on the model of the LA created with the electroanatomic mapping system. At the tagged sites, RF energy was applied until the maximum local electrogram amplitude decreased by $\geq 50 \%$ or to $<0.1 \mathrm{mV}$ ?

After the LACA, the presence of PV potentials was examined with a 7-Fr decapolar ring catheter (Lasso, Biosense Webster) positioned at the PV ostium5,6 In the presence of PV potentials within the PV, PV isolation was performed as previously described, $6,16,17$ The Lasso catheter was positioned inside the PV, within $5 \mathrm{~mm}$ of the ostium. For optimal separation of the PV potentials from the atrial electrograms, mapping and ablation in the left superior and inferior PV were performed during distal coronary sinus pacing at a cycle length of $600 \mathrm{~ms}, 5,6,16,17$ The target sites for ablation were selected by identifying the PV potentials that had an equivalent or earlier activation relative to the adjacent Lasso catheter recording sites. The ablation catheter was always positioned in close proximity and on the ostial side of the Lasso catheter. RF ablation was performed using a maximum power of $30 \mathrm{~W}$ and a maximum electrode-tissue interface temperature of $52-55^{\circ} \mathrm{C}^{3,4}$ The applications of energy were 60-90 s in duration. If AF still was present after the LACA and PV isolation, transthoracic cardioversion was performed. In 7 patients, a linear ablation at the cavo-tricuspid isthmus was also performed for typical atrial flutter ${ }^{8}$

After the ablation procedure, heparin was infused for a few days, at which point the patients were treated with warfarin for 2 to 3 months and class I and/or III antiarrhythmic drugs for the first 3 months after the ablation. When there was a recurrence of $\mathrm{AF}$ in the hospital, an additional 


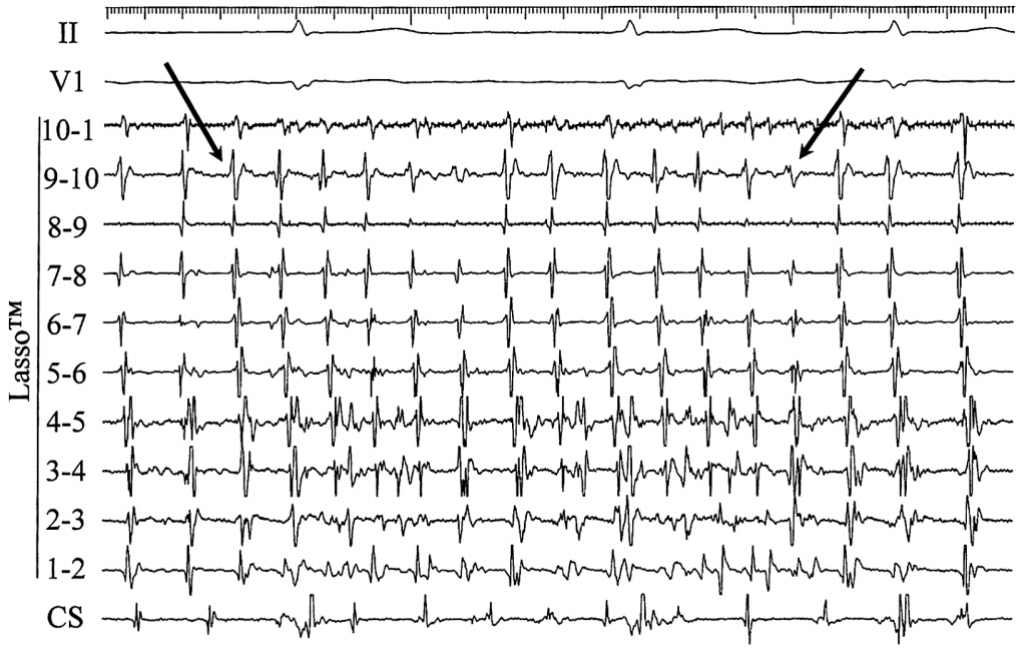

Stim
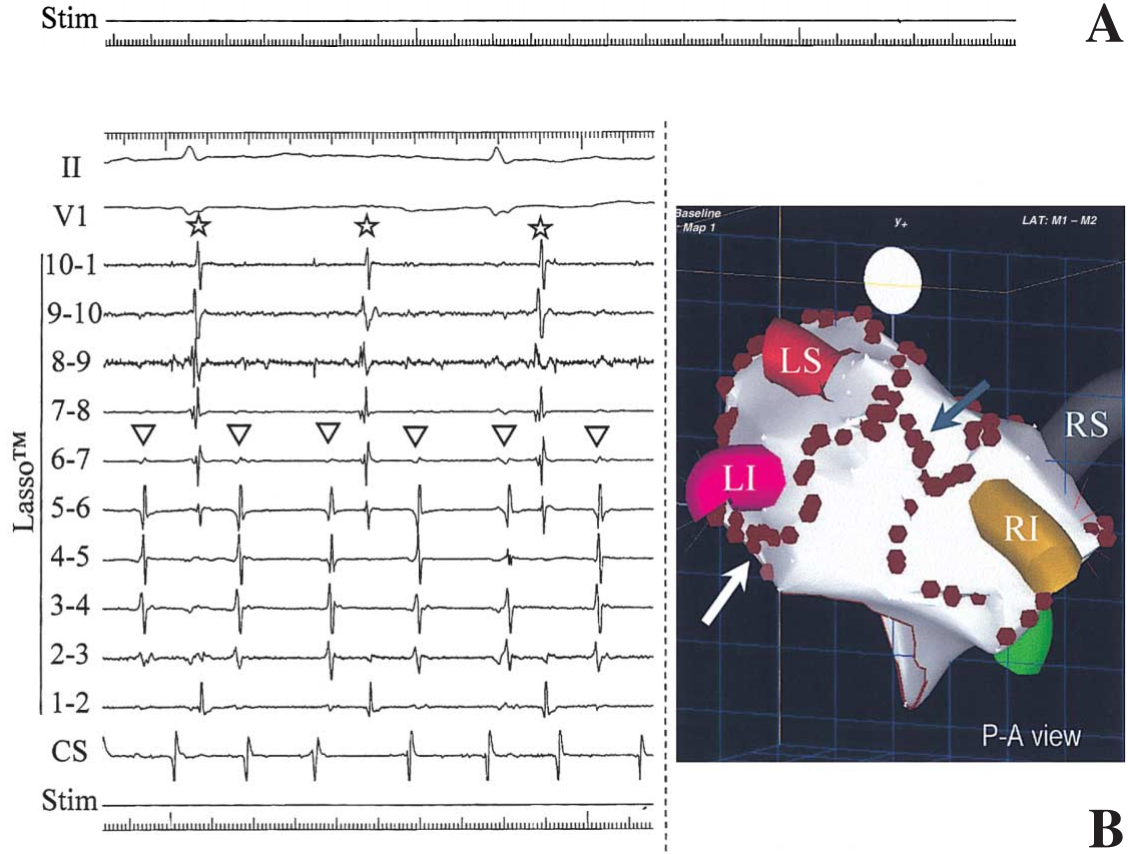

Fig 2. An example of a left atrial and pulmonary vein $(\mathrm{PV})$ ostial ablation before and after the ablation procedure. (A) Before ablation. Shown are leads II and $\mathrm{V}_{1}, 10$ bipolar electrograms recorded by a Lasso catheter $\left(\mathrm{Lasso}^{\mathrm{TM}}\right)$ positioned in the left superior PV and a bipolar electrogram recorded in the coronary sinus (CS). A complex activation pattern was recorded in this vein. The arrows mark the onset and offset of an intermittent burst of tachycardia, which had a duration of $1,600 \mathrm{~ms}$. The mean cycle length of the PV tachycardia was $122 \mathrm{~ms}$ and the simultaneous mean cycle length in the CS was $164 \mathrm{~ms}$. (B) After left atrial catheter ablation. Intracardiac electrograms demonstrated that 2 fascicles activated simultaneously with different cycle lengths of $410 \mathrm{~ms}$ (asterisks) and $220 \mathrm{~ms}$ (open triangles), respectively, which were longer than those before the ablation (Left panel). The right panel shows the ablation lines created during left atrial catheter ablation. A 3-dimensional representation of the left atrium and PV was constructed with an electroanatomic mapping system. The red tags represent the sites at which the radiofrequency energy was delivered. The leftand right-sided PV are encircled. Also shown are the ablation lines at the mitral isthmus (white arrow) and posterior left atrium (gray arrow). (C) After segmental isolation of the PV. No PV potentials were recorded after 2 applications of radiofrequency energy at the sites where the earliest activation was recorded by a Lasso catheter (electrodes 5 and 9) within this vein. The radiograms show the ablation catheter and Lasso catheter positioned near the ostium of this vein (Right panels). The ablation catheter $(\mathrm{Abl})$ was positioned near the ostium of this vein where the earliest activation was recorded by a Lasso catheter (electrode 5). L(R)AO, left (right) anterior oblique projection; LI(S), left inferior (superior) pulmonary vein; RI(S), right inferior (superior) pulmonary vein; Stim, stimulus. 
antiarrhythmic drug was added. After the ablation procedure, the patients underwent follow-up ( 2 weeks after the procedure, then every 1-2 months at our cardiology clinic). To confirm the absence of AF, a 12-lead ECG was recorded on each visit to the clinic, and 24-h Holter monitoring was also used at least once during the follow-up period. The recurrence of AF was defined as recurrence of symptoms suggestive of tachycardia and an episode of AF documented on the 12-lead ECG or 24-h Holter monitoring during the follow-up period. About 3 months later, discontinuation of the antiarrhythmic drugs was attempted in the patients who did not have any symptoms suggestive of AF recurrence or frequent premature atrial contractions (PAC). A stepwise reduction in the dosage of the antiarrhythmic drug or discontinuation of one of the antiarrhythmic drugs when 2 were given, was attempted. In contrast, in the patients who had an $\mathrm{AF}$ recurrence or frequent PAC, the antiarrhythmic drug treatment was continued. Narrowing of the PV was examined using a computed tomography scan 2-6 months after the ablation procedure.

\section{Results}

\section{LACA}

The mean number of minutes of RF energy required to encircle the PV was $17 \pm 4$ for the left-sided PV and $11 \pm 3$ for the right-sided PV. LACA was able to be performed in all patients, and the mean total duration of RF energy applications for the entire LACA procedure was $40 \pm 9 \mathrm{~min}$.

\section{PV Isolation}

After LACA, 38 PV (59\%) were completely isolated, and complete PV isolation could be achieved with only a few RF energy applications $(2.7 \pm 2.0 \mathrm{~min})$ at the narrow area of the PV ostium $(24 \pm 15 \%)$ in the remaining PV (Table 1; Fig 2). Additional RF applications to isolate the $\mathrm{PV}$ were required more often in the superior $\mathrm{PV}$ than in the inferior PV $(\mathrm{p}<0.001$; Table 1). However, complete isolation of the PV could be achieved with RF energy applications at the ostium in 62 of 64 (97\%) targeted PV.

\section{Resolution of AF and the Total Procedure Time}

Sinus rhythm was restored during the ablation procedure in 4 patients $(25 \%)$, while external cardioversion (200 J) was required to restore sinus rhythm in the remaining 12 patients $(75 \%)$. The mean total duration of the procedure, including the procedure time for the ablation of the atrial flutter at the cavo-tricuspid isthmus in 7 patients, was $200 \pm 39 \mathrm{~min}$. Fifteen patients were treated with antiarrhythmic drugs after the procedure (pirmenol-2; pilsicainide-1; flecainide-2; amiodarone-8, pirmenol + amiodarone-1; and pilsicainide + amiodarone-1), and the remaining patient had no antiarrhythmic drugs because the patient had a history of side effects from various drugs and refused antiarrhythmic drug treatment.

\section{Recurrence of AF After the Ablation Procedure}

AF recurred within $9 \pm 11$ days after the procedure (range, 1-26 days) in 7 patients (44\%). In 2 patients who had recurrent AF 2 days after the procedure, a repeat ablation procedure was performed. A PV tachycardia originating from the right superior PV (RSPV) ostium $(n=1)$ or left inferior PV ostium $(n=1)$, which was located at a more proximal site to where the $\mathrm{PV}$ isolation had been performed in the PV in the initial ablation procedure, were found and successfully ablated. Recovery of conduction in a PV that had been previously isolated was found in the RSPV in the former patient and in the RSPV, right inferior PV and left superior PV in the latter patient. In both patients, complete isolation of all targeted PV was achieved after the second procedure.

In 1 patient who developed AF 23 days after the ablation, sinus rhythm was restored with an intravenous infusion of $70 \mathrm{mg}$ of cibenzoline, and bepridil $(100 \mathrm{mg} /$ day $)$ was added to the treatment with flecainide $(150 \mathrm{mg} /$ day $)$. In the remaining 4 patients, sinus rhythm could not be maintained despite the use of amiodarone $(n=4)$ and/or external cardioversion $(\mathrm{n}=2)$. The patients who could not maintain sinus rhythm had a significantly larger LA $(49 \pm 5 \mathrm{~mm})$ than those who were in sinus rhythm $(43 \pm 4 \mathrm{~mm} ; \mathrm{p}<0.05)$. Two patients with a recurrence of AF had mitral stenosis, with LA diameters of $48 \mathrm{~mm}(\mathrm{n}=1)$ and $56 \mathrm{~mm}(\mathrm{n}=1)$, respectively. No patients had a recurrence of AF $>1$ month after the ablation procedure.

Approximately 3 months after the procedure, attempts to stop the antiarrhythmic drug treatment were attempted in 11 patients in whom sinus rhythm could be maintained with antiarrhythmic drugs. The antiarrhythmic drug treatment was successfully stopped in 1 patient. However, treatment with antiarrhythmic drugs had to be continued with the same dosage as at the time of discharge $(n=4)$ or with a reduction in the total dosage of the antiarrhythmic drugs $(n=6)$ because frequent PAC were documented on the 12lead ECG or 24-h Holter monitoring. Alternatively, symptoms suggestive of frequent PAC continued at that time or appeared after the reduction in the drug dosage. At the end of the follow-up period of 17 \pm 3 months (range, 12-22 months), 12 patients $(75 \%)$ maintained normal sinus rhythm and were free of symptomatic AF with $(n=10$; pirmenol-1; pilsicainide-1; flecainide-1; amiodarone-6, and pirmenol + amiodarone-1) or without antiarrhythmic drugs $(\mathrm{n}=2)$, and $9(75 \%)$ had a history of recurrent AF with external cardioversion and antiarrhythmic drugs (Fig 1B).

\section{Complications}

A cerebral infarction occurred just after the ablation procedure in 1 patient, and he demonstrated left homonymous hemianopia during the follow-up period. In the remaining 15 patients, no potential complications occurred during the isolation or ablation procedures. No patients complained of symptoms suspicious of PV stenosis or an occlusion, and no narrowing of a PV greater than $50 \%$ was detected on the computed tomography scan 2-6 months after the ablation procedure in all patients.

\section{Discussion}

\section{Major Findings}

The results of the present study demonstrated that LACA with complete isolation of all $4 \mathrm{PV}$ was effective in maintaining sinus rhythm in patients with drug-resistant, persistent AF. Although the incidence of recurrent AF after the ablation procedure was relatively high, a tailored treatment, such as a repeat ablation procedure, changes in the treatment with antiarrhythmic drugs and/or the use of the amiodarone were effective in the restoration and/or maintenance of sinus rhythm in most of these cases. As a result, in $75 \%$ of the patients, sinus rhythm was maintained at the end of the mid-term follow-up period. The patients in whom sinus rhythm could not be maintained had larger LA 
than those who were in sinus rhythm. Stroke occurred just after the procedure in 1 patient, but no other complications, including PV stenosis, were found during the follow-up period. These results may indicate that although treatment with antiarrhythmic drugs is needed in most patients, this new approach is an effective treatment for persistent AF.

Proposed Mechanisms of the Maintenance of Sinus Rhythm

Recently, the LACA technique has been reported as an effective treatment for PAF. The LA ablation technique used in the present study may have effects that are helpful in preventing $\mathrm{AF}$, and those are: (1) creating circumferential lesions around the PV may isolate the non-PV triggers (eg, the posterior LA and vein of Marshall) as well as PV triggers of $\mathrm{AF} ; 19,20$ (2) the ablation lines may eliminate the anchor points for rotors or mother waves that drive AF;21,22 (3) the ablation line that connects the 2 encircling ablation lines may eliminate sources of $\mathrm{AF}$ that arise from the posterior wall of the LA;3,19 (4) approximately $25 \%$ to $30 \%$ of the LA myocardium is excluded by the encircling lesions, ${ }^{10}$ thereby limiting the area available for circulating wavelets that may be needed to perpetuate $\mathrm{AF} ; 23$ and (5) LA ablation may attenuate vagal reflexes, rendering the atrium less vulnerable to $\mathrm{AF}^{24}$ These effects of LACA are incremental to the effects of segmental ostial ablation and may account for the greater efficacy of LACA in eliminating persistent $\mathrm{AF}$, as well as PAF? From those reasons, the strategy of combining the LACA and PV isolation techniques performed in the present study might also be effective for persistent AF.

It still remains unclear whether or not the electrical isolation of the PV after LACA is indispensable for curing persistent AF. In PAF, it is considered that PV play a critical role in initiating and maintaining $\mathrm{AF}^{1-5}$ In persistent $\mathrm{AF}$, it is possible that the electrophysiological and anatomic remodeling that occurs during persistent $\mathrm{AF}$ often allows the atria to continue the fibrillation independently of the PV5 Therefore, in persistent AF, PV may play a less critical role in maintaining AF than in PAF5 However, recent studies demonstrated the importance of the electrical isolation of the PV for curing chronic AF in patients with mitral valvular disease who underwent artificial valve replacements 25,26 Therefore, we thought that the creation of a complete PV isolation after the LACA was also important for eliminating persistent $\mathrm{AF}$.

An ablation catheter with an 8-mm distal electrode was used for the LACA procedure in the previous study, 10 However, in the present study, we used an ablation catheter with a 4-mm distal electrode. This might carry the risk of an incomplete PV isolation after the LACA or early restoration of the conduction between the LA and PV after the creation of a complete PV isolation as compared with the use of a catheter with an 8-mm distal electrode. In fact, after the LACA procedure, complete PV isolation could not be obtained of most PV, especially of the superior PV. Therefore, we think that confirmation of a complete isolation of the PV and creation of a complete PV isolation after the LACA procedure is important when an ablation catheter with a 4-mm distal electrode is used for the LACA procedure.

\section{Previous Studies}

Electrical cardioversion has been used as an established method for treating persistent $\mathrm{AF}$, with a primary success rate of up to $95 \% ?^{27-32}$ However, the long-term results for the maintenance of sinus rhythm after electrical cardioversion are discouraging: Sinus rhythm was able to be maintained in only $13-25 \%$ of patients without prophylactic antiarrhythmic therapy,29,30 and in only $25-68 \%$ of those with antiarrhythmic therapy $31,32 \mathrm{PV}$ isolation was expected to be a curative treatment for drug-resistant, persistent AF. However, only $29 \%$ of the patients with persistent AF were either free of symptomatic AF or markedly improved at the 5 month follow-up, which was consistent with a previous study in which PV isolation was performed intraoperatively under direct visualization in patients with chronic $\mathrm{AF}$ where sinus rhythm was restored in only $\approx 33 \%$ of patients 33

Several studies have reported the effectiveness of the LACA for drug-resistant AF, 10,27,28 However, the site where the linear ablation was performed in the LA differed and the utility of the LACA procedure in persistent AF has not been sufficiently determined, 10,27,28 Recently, Hsu et al reported the effectiveness of a combined approach of LACA and PV isolation in restoring and maintaining sinus rhythm in patients with persistent $\mathrm{AF}^{28}$ Their strategy for the ablation technique was a PV isolation followed by the creation of 1 or more linear lesions bridging the 2 superior PV and/or extending a line from a PV to the mitral annulus $2^{28}$ Although the ablation strategy differed from the present study, and the follow-up period after the ablation procedure was shorter than in the present study, about $80 \%$ of the patients remained in sinus rhythm after the ablation procedure28 In the present study, with the new strategy of combining the LACA and PV isolation techniques, sinus rhythm could also be maintained in $75 \%$ of the patients with drug-resistant, persistent $\mathrm{AF}$, and $75 \%$ of them had a history of having failed to maintain sinus rhythm after electrical cardioversion and prophylactic antiarrhythmic drugs. The results from the present study and a previous study demonstrated that a combined approach using LACA and $\mathrm{PV}$ isolation may be effective in persistent $\mathrm{AF}$ as well as $\mathrm{PAF}^{28} \mathrm{We}$ believe that LACA with electrical isolation of all $4 \mathrm{PV}$ is crucial for maintaining sinus rhythm after the ablation procedure.

PV stenosis is one of the potential complications after the PV isolation. RF energy delivery in the PV, even in the vicinity of the ostia, is associated with the development of PV stenosis, and a greater amount of RF energy delivered at the PV ostium results in a narrower PV 7,8 Therefore, it is crucial to deliver the RF energy within the PV as seldom as possible to avoid PV stenosis. In the present study, the PV isolation following the LACA could be performed with less RF energy deliveries and a narrower ablated area within the $\mathrm{PV}$, as compared with the results of the PV isolation reported previously 34,35 As a result, no significant PV stenosis occurred in any patients. Therefore, this technique seems to be effective for avoiding lethal PV stenosis, however, longterm follow-up of the PV narrowing could not be performed at present.

\section{Study Limitations}

First, our sample size was relatively small and antiarrhythmic drugs were used in most patients after the procedure, which might obscure the effectiveness of this technique. However, in the present study, sinus rhythm could be maintained in $75 \%$ of the patients during the $17 \pm 3$ months of follow-up period, which was longer than that in the previous studies, and might imply that our findings are somewhat robust ${ }^{2}{ }^{8}$ However, to clarify the long-term effect of this treatment on the patients with persistent $\mathrm{AF}$, future 
studies with a larger number of patients and without the use of concomitant antiarrhythmic drugs may be needed.

Finally, esophageal fistulas have been raised as a potential complication after LACA with an 8-mm tip ablation catheter.11 Because the esophagus is located just behind the LA, and both the esophageal and LA posterior walls are quite thin, ${ }^{36} \mathrm{RF}$ energy applications at the LA posterior wall might result in this lethal complication.1 ${ }^{1}$ In the present study, RF energy was limited to a maximum of $35 \mathrm{~W}$ when the LA posterior wall was ablated, and the ablation was performed with a 4-mm tip catheter. However, no conclusions could be drawn about how much energy was safe in order to avoid a fistula formation or whether or not the RF energy delivery from the 4-mm tip catheter was safer than that from an 8-mm tip catheter. Therefore, as recently reported, imaging of the esophagus by swallowing a radiocontrast agent or placing a small-caliber probe into the esophagus before the ablation procedure may be important for confirming the relationship between the LA and esophagus and for avoiding an RF energy delivery to the LA posterior wall adjacent to the esophagus!1

\section{Conclusions}

A combined approach of LACA and PV isolation with or without antiarrhythmic drugs was effective in restoring and maintaining sinus rhythm in patients with drug-refractory, persistent AF.

\section{Acknowledgments}

The present was supported by a research grant for Cardiovascular Diseases (14C-2) from the Ministry of Health, Labor and Welfare, Japan. We are indebted to Ms Rika Utsugi and Mr Tsutomu Nakajima for their important contribution to the present study.

\section{References}

1. Häissaguerre M, Jäis P, Shah DC, Takahashi A, Hocini M, Quiniou $\mathrm{G}$, et al. Spontaneous initiation of atrial fibrillation by ectopic beats originating in the pulmonary veins. N Engl J Med 1998; 339: 659666.

2. Oral H, Ozaydin M, Tada H, Chugh A, Scharf C, Hassan S, et al. Mechanistic significance of intermittent pulmonary vein tachycardia in patients with atrial fibrillation. $J$ Cardiovasc Electrophysiol 2002; 13: $645-650$.

3. Häissaguerre M, Shah DC, Jäis P, Hocini M, Yamane T, Deisenhofer I, et al. Electrophysiological breakthroughs from the left atrium to the pulmonary veins. Circulation 2000; 102: 2463-2465.

4. Morady F. Treatment of paroxysmal atrial fibrillation by pulmonary vein isolation. Circ J 2003; 67: 567-571.

5. Oral H, Knight BP, Tada H, Özaydin M, Chou A, Hassan S, et al. Pulmonary vein isolation for paroxysmal and persistent atrial fibrillation. Circulation 2002; 105: 1077-1081.

6. Tada H, Naito S, Kurosaki K, Ueda M, Ito S, Shinbo G, et al. Segmental pulmonary vein isolation for paroxysmal atrial fibrillation improves quality of life and clinical outcomes. Circ J 2003; 67: 861 865 .

7. Saad EB, Rossillo A, Saad CP, Martin DO, Bhargava M, Erciyes D, et al. Pulmonary vein stenosis after radiofrequency ablation of atrial fibrillation: Functional characterization, evolution, and influence of the ablation strategy. Circulation 2003; 108: 3102-3107.

8. Dill T, Neumann T, Ekinci O, Breidenbach C, John A, Erdogan A, et al. Pulmonary vein diameter reduction after radiofrequency catheter ablation for paroxysmal atrial fibrillation evaluated by contrast-enhanced three-dimensional magnetic resonance imaging. Circulation 2003; 107: $845-850$.

9. Oral H, Scharf C, Chugh A, Hall B, Cheung P, Good E, et al. Catheter ablation for paroxysmal atrial fibrillation: Segmental pulmonary vein ostial ablation versus left atrial ablation. Circulation 2003; 108: 2355-2360.

10. Pappone C, Rosanio S, Oreto G, Tocchi M, Gugliotta F, Vicedomini $\mathrm{G}$, et al. Circumferential radiofrequency ablation of pulmonary vein ostia: A new anatomic approach for curing atrial fibrillation. Circulation 2000; 102: 2619-2628.

11. Pappone C, Oral H, Santinelli V, Vicedomini G, Lang CC, Manguso $\mathrm{F}$, et al. Atrio-esophageal fistula as a complication of percutaneous transcatheter ablation of atrial fibrillation. Circulation 2004; 109: $2724-2726$.

12. Ouyang F, Antz M, Ernst S, Hachiya H, Mavrakis H, Deger FT, et al. Recovered pulmonary vein conduction as a dominant factor for recurrent atrial tachyarrhythmias after complete circular isolation of the pulmonary veins: Lessons from double Lasso technique. Circulation 2005; 111: $127-135$.

13. Lemola K, Hall B, Cheung P, Good E, Han J, Tamirisa K, et al. Mechanisms of recurrent atrial fibrillation after pulmonary vein isolation by segmental ostial ablation. Heart Rhythm 2004; 1: 197-202.

14. Tada H, Ito S, Naito S, Yamada M, Miyaji K, Hashimoto T, et al. Left atrial and pulmonary vein ostial catheter ablation followed by the linear ablation at the cavo-tricuspid isthmus restored sinus rhythm in a patient with persistent atrial fibrillation. Shinzo 2005; (in Japanese) (in press).

15. Ho SY, Sanchez-Quintana D, Cabrera JA, Anderson RH. Anatomy of the left atrium: Implications for radiofrequency ablation of atrial fibrillation. J Cardiovasc Electrophysiol 1999; 10: 1525-1533.

16. Tada H, Oral H, Knight BP, Özaydin M, Chou A, Scharf C, et al. randomized comparison of bipolar vs unipolar plus bipolar recordings during segmental ostial ablation of pulmonary veins. $J$ Cardiovasc Electrophysiol 2002; 13: 851-856.

17. Tada H, Oral H, Greenstein R, Pelosi F, Knight BP, Strickberger SA, et al. Differentiation of atrial and pulmonary vein potential recorded circumferentially within pulmonary veins. $J$ Cardiovasc Electrophysiol 2002; 13: 118-123.

18. Tada H, Oral H, Sticherling C, Chough SP, Baker RL, Wasmer K, et al. Double potentials along the ablation line as a guide to radiofrequency ablation of typical atrial flutter. J Am Coll Cardiol 2001; 38: $750-755$.

19. Lin WS, Tai CT, Hsieh MH, Tsai CF, Lin YK, Tsao HM, et al. Catheter ablation of paroxysmal atrial fibrillation initiated by non-pulmonary vein ectopy. Circulation 2003; 107: 3176-3183.

20. Hwang C, Wu TJ, Doshi RN, Peter CT, Chen PS. Vein of marshall cannulation for the analysis of electrical activity in patients with focal atrial fibrillation. Circulation 2000; 101: 1503-1505.

21. Jalife J. Rotors and spiral waves in atrial fibrillation. $J$ Cardiovasc Electrophysiol 2003; 14: 776-780.

22. Jalife J, Berenfeld O, Mansour M. Mother rotors and fibrillatory conduction: A mechanism of atrial fibrillation. Cardiovasc Res 2002; 54: $204-216$.

23. Moe GK. A conceptual model of atrial fibrillation. J Electrocardiol 1968; 1: 145-146.

24. Pappone C, Santinelli V, Manguso F, Vicedomini G, Gugliotta F, Augello G, et al. Pulmonary vein denervation enhances long-term benefit after circumferential ablation for paroxysmal atrial fibrillation. Circulation 2004; 109: 327-334.

25. Sueda T, Imai K, Ishii O, Orihashi K, Watari M, Okada K. Efficacy of pulmonary vein isolation for the elimination of chronic atrial fibrillation in cardiac valvular surgery. Ann Thorac Surg 2001; 71: $1189-1193$.

26. Tada H, Ito S, Naito S, Hasegawa $\mathrm{Y}$, Kurosaki K, Ezure M, et al. Simple cryoablation with a special made cryoprobe for eliminating chronic atrial fibrillation associated with mitral valve disease: Longterm outcome. Pacing Clin Electrophysiol 2005; 28: S73-S77.

27. Ouyang F, Bansch D, Ernst S, Schaumann A, Hachiya H, Chen M, et al. Complete isolation of left atrium surrounding the pulmonary veins: New insights from the double-Lasso technique in paroxysmal atrial fibrillation. Circulation 2004; 110: 2090-2096.

28. Hsu LF, Jais P, Sanders P, Garrigue S, Hocini M, Sacher F, et al. Catheter ablation for atrial fibrillation in congestive heart failure. $N$ Engl J Med 2004; 351: 2373-2383.

29. Coplen SE, Antman EM, Berlin JA, Hewitt P, Chalmers TC. Efficacy and safety of quinidine therapy for maintenance of sinus rhythm after cardioversion: A meta-analysis of randomized control trials. Circulation 1990; 82: 1106-1116.

30. Lehto M, Kala R. Persistent atrial fibrillation: A population based study of patients with their first cardioversion. Int J Cardiol 2003; 92: $145-150$.

31. Wanless RS, Anderson K, Joy M, Joseph SP. Multicenter comparative study of the efficacy and safety of sotalol in the prophylactic treatment of patients with paroxysmal supraventricular tachyarrhythmias. Am Heart J 1997; 133: 441 - 446.

32. Katritsis DG, Panagiotakos DB, Karvouni E, Giazitzoglou E, Korovesis S, Paxinos G, et al. Comparison of effectiveness of carvedilol versus bisoprolol for maintenance of sinus rhythm after 
cardioversion of persistent atrial fibrillation. Am J Cardiol 2003; 92: $1116-1119$.

33. Melo J, Adragao P, Neves J, Ferreira MM, Pinto MM, Rebocho MJ, et al. Surgery for atrial fibrillation using radiofrequency catheter ablation: Assessment of results at one year. Eur J Cardiothorac Surg 1999; 15: 851-854.

34. Kurosaki K, Tada H, Naito S, Ueda M, Ito S, Shinbo G, et al. Significance and efficacy of repeat ablation procedures in patients with recurrent atrial fibrillation after segmental isolation of the pulmonary veins. J Arrhythmia 2004; 20: 39-45 (in Japanese).

35. Kurosaki K, Tada H, Naito S, Ueda M, Ito S, Shinbo G, et al. Incidence and clinical characteristics of delayed success after segmental isolation of the pulmonary veins in patients with paroxysmal atrial fibrillation. J Arrhythmia 2004; 20: 482 -489 (in Japanese).

36. Lemola K, Sneider M, Desjardins B, Case I, Han J, Good E, et al. Computed tomographic analysis of the anatomy of the left atrium and the esophagus: Implications for left atrial catheter ablation. Circulation 2004; 110: 3655-3660. 